\title{
Two Kinds of Theory: What Psychology Can Learn from Einstein.
}

\section{Marek McGann ${ }^{1}$ and Craig P. Speelman ${ }^{2}$}

${ }^{1}$ Department of Psychology, Mary Immaculate College, Limerick. Email: marek.mcgann@mic.ul.ie

${ }^{2}$ School of Psychology \& Social Science, Edith Cowan University

\begin{abstract}
A century ago, Einstein (1919) distinguished between two kinds of theory - theories of principle, and constructive theories. These have separate but complementary roles to play in the advancement of knowledge, in the manner in which they relate to data, and how they are developed. The different kinds of theory carry implications for what kinds of data we produce, and how they are put to use. We outline Einstein's distinction and the model of theory formation that it involves. We then use the distinction to look at some of the discussion of scientific practice in psychology, particularly recent work on the need for more theoretical, rather than purely methodological, sophistication. We argue in agreement with Einstein that the distinction is a useful one, and that adopting it as a tenet of theoretical development requires a renewed commitment to a natural history of psychology.
\end{abstract}

Keywords: scientific practice, theories of principle, constructive theories, natural history, exploratory research

\section{Introduction}

Within the broad discussion on scientific practice in psychological research currently underway there is an increasingly explicit recognition that not just our methods, but also our theories need a greater degree of sophistication. Szollosi and Donkin (2019a) note that recent discussion of the theoretical aspects of reforms to scientific practice was at first relegated to less formal channels of communication (commentaries and blogposts, such as Borsboom, 2013; Carsel, Demos, \& Motyl, 2018; Seymour, 2019; van Rooij, 2019; Wilson, 2011), but is now increasingly represented in preprint and traditional article publications (Gray, 2017; Muthukrishna \& Heinrich, 2019; Oberauer \& Lewandowsky, 2019; Szollozi \& Donkin, 2019a,b). Criticisms of theory development in psychology are nothing new - there is a decades long tradition of it (Dar, 1987; Dewsbury, 2009; Gigerenzer, 1998; Lykken, 1991; Meehl, 1978). The present discussion, embedded as it is within the broader considerations of professional reforms, however, carry a weight that previous efforts have appeared to lack. The literature on these considerations of theory at present tend to focus on their variability (Szollosi \& Donkin, 2019a,b), logical coherence (Oberauer \& Lewandowsky, 2019; Muthukrishan \& Heinrich, 2019) and adequate explication (Gray, 2017). We agree with these arguments. The characteristics of theories affect the ways in which data carry implications for them, and this in

This is the accepted text. Full paper in press at Theory \& Psychology (2020)

DOI:

Correspondence Marek McGann

(c) Marek McGann \& Craig Speelman 2020 turn affects how we should form hypotheses, what data we generate to test them, and what kinds of techniques we use to interpret those data.

In the rest of this paper we argue that a valuable addition to this reflection on theory would be to step back and examine what kinds of theory there are. Theories do not form a homogeneous category. Rather, there are different kinds of theories which can be deployed in science for distinct purposes, and from which we draw implication for our understanding and valuing of various methods and the data that they produce. To illustrate this theoretical diversity we introduce a simple distinction 
first described explicitly by Einstein, and explore some of the implications of this distinction, and recommendations that follow from it for the improvement of psychological research practices.

\section{Two Kinds of Theory}

In 1919, in a letter to the Times of London, Albert Einstein distinguished between two different kinds of scientific theory. He claimed that most are "constructive theories", in which models of reality are constructed from simple propositions concerning hypothesized components. Einstein's example here was the kinetic theory of gases, which explains the measured temperature and pressure of gases in a container on the basis of the movements of molecules of the gas. We understand a phenomenon when we have a constructive theory which encompasses it, and the theory enables an effective coordination of our actions with the phenomena in question (accurate prediction, and perhaps some form of control). We can see that insofar as this is the case, then the development of effective, useful, and comprehensive constructive theory is the proper work of a mature science.

In psychological research, when theorizing is done explicitly it tends to be of this kind, focusing on hypothesized components and the means by which they might give rise to behavior observed in controlled conditions. The repudiation of a simplistic behaviorist attitude that many of us learn in our undergraduate studies ensures that from the beginning of our careers the target of our science is the adequate description of mechanisms that are typically not directly observable or measurable, but which underlie things observed and measured.

The second form of theory described by Einstein is what he termed "theories of principle". Theories of principle are those generalizations from observations that are elegant, accurate, and useful descriptions of the natural world captured in those observations. Einstein (1919) notes that Newton's theory of universal gravitation and his own theories of special and general relativity fall into this category. Theories of principle do not provide accounts of mechanisms. They do not begin from a specified set of hypothesized components, but rather are developed on the basis of abductively identified principles. From these principles, then, descriptions of other key facets of the world can be derived. From a diversity of observations the scientist conjectures key relationships that characterize the phenomena of interest, and with those conjectures fixed as principles, further details can be analytically deduced (Holton, 1979). They are effective insofar as they are pure, logical, and secure in their foundation in empirical data (Einstein, 1919).

The special and general theories of relativity fix the speed of light, not on the basis of an explanation for it, but multiple empirical demonstrations. Having identified this fact as one of a set of basic principles, however, Einstein was able to get a surprising amount of work done in describing relationships between phenomena in the universe without offering any mechanistic account of how those relationships arise. The theory of relativity, for instance, famously predicts changes in mass and a slowing of time as bodies accelerate, limited by the speed of light. There is no mention made of the means by which mass and time change. Their relationship is captured by the theory in a way that has allowed for more than a century of accurate predictions, with no account as to precisely how it all works. There is no equivalent in relativity to the kinetic theory of gases, which explains how temperature and pressure are produced via the movements of molecules. The "how" of relativity remains an outstanding challenge for physicists, but it is a challenge with very clearly defined parameters.

The variant characteristics of these two kinds of theory give them different strengths and different roles to play in the development of our knowledge. A better understanding of these differences 
will help us see both the value of them, and the implications that they hold for how we go about producing data to inform them.

\subsection{Complementary theories supporting advancement of knowledge}

Felline (2011) describes theories of principle as capturing the "structural" aspects of the phenomena of interest. That is to say that theories of principle provide accounts of the relationships and patterns that exist in descriptions of the world, rather than descriptions of the mechanisms that give rise to those relationships. They offer these descriptions, however, in the formal terms of the scientific discipline (in Einstein's case, those terms are substantially mathematical). The perception and understanding of these patterns might arise from extensive observations and tabulations of data, introducing new concepts and ways of thinking that help organize existing observations (as in the case of Newton's theory of universal gravitation). They might re-orient the scientific community around these new patterns, in response to challenges or confusions regarding them (as was the case with Einstein's theory of relativity). In focusing on the structural aspects of things, theories of principle make few ontological commitments (or as few as possible). Some (perhaps Einstein included; Balashov \& Jansson, 2003, p.332) consider theories of principle to be the inferior form of theory - they provide description more than explanation. But they still have a powerful role to play in scientific endeavor.

Firstly, they offer what Felline (2011) calls "structural explanations", models which explain the behavior of some part of the system in terms of its relationships to other parts of the model, without necessarily specifying how those relationships arise from the properties of the parts themselves (see also Lange, 2014). Secondly, having identified the principles on the basis of patterns in the observed phenomena, predictions that follow from those principles can be deduced. Indeed, Einstein's letter to the Times was prompted by the so-called Eddington Experiment. The Eddington Experiment, organized by Arthur Stanley Eddington and Frank Watson Dyson, involved measurements of starlight deflection made during a solar eclipse on either side of the Atlantic (Brasil on the west and Principe on the east). The measurements were to test predictions made by Einstein as early as 1911, and which were posited as direct tests of general relativity in 1915. A final, and perhaps most important role for theories of principle is in specifying the relationships between chosen components of an observed system in the formal terms of the discipline, theories of principle impose constraints on constructive theories (Flores, 1999; Einstein 1919).

If the theory of principle is a good one, then any constructive theory in the domain will have to encompass it, and should not make any predictions that violate those principles. In this way, the theory of principle becomes the representative of natural settings within experimental laboratory work. The theory of principle can help identify research questions, set research agendas, and by setting constraints, support the evaluation of competing constructive theories. Einstein's own theory of general relativity has performed such functions within the domain of cosmological physics for the better part of a century (once it came to be accepted by the scientific community at large).

Good theories of principle are therefore somewhat the more stable of the two kinds, not being beholden to the specific ontological or mechanistic commitments required of constructive theories (Felline, 2011; Balashov \& Jansson, 2003, suggest this is partly what drew Einstein to the form of theory which he considered technically inferior). Constructive theories, however, provide descriptions and explanations at a higher degree of resolution more in tune with the highest aspirations of science. Bluntly summarizing, Balashov and Jansson (2003) claim that constructive theories are about the underlying reality, while theories of principle are about the phenomena.

McGann \& Speelman | This is the accepted text. Full paper in press at Theory \& Psychology 
This complementary relationship between theories of principle and constructive theories offers a division of labor in science that can play an important role in structuring and coordinating diverse research agendas. Theories of principle, where shown to be accurate in their descriptions of the world, provide an agreed set of criteria by which otherwise varied constructive approaches can be assessed, an arena within which they can be compared directly. The theory of principle is the formalized surrogate of the natural world used to inform and evaluate studies in controlled environments.

In having different roles to play in scientific practice, the different kinds of theory have somewhat different criteria by which they should be judged, and also, crucially, different relationships to data. It is this latter aspect in particular that we argue holds some important implications for the priorities of contemporary psychological research.

\subsection{Different kinds of theories have different relationships with data}

As noted above, Einstein describes theories of principle as being evaluated by the soundness of their logic, coherence, and the security of their foundation in data. This foundation in data is worth dwelling on for a moment.

Einstein describes the principles being first identified in a conjecture born of familiarity with the "multiplicity" of sense experience. Theories of principle are thus developed abductively. The data which inspire such leaps of intuition are varied, general. Einstein wants to, as much as possible, encompass the "totality of sense experiences" (Holton, 1979, p.313; see also Einstein in Schilpp, 1949). The variety and generality of these data are crucial. For the integrating conjecture that is vital to Einstein's formation of a theory to have validity, it must attempt to encompass this totality.

We have already noted that a theory of principle should make as few ontological commitments as possible. Though observations or measurements that are wholly theory-free are impossible, each prior theoretical commitment means that a constructive theory (whether implicit or explicit) already plays a role in its structure. Each such commitment narrows the field of experience that can possibly be encompassed by the theory when developed. Laboratory practice is perforce structured by prior theory - every decision made with regard to method, material, and measurement, represents the researcher's informed perspective. Opportunities for serendipity and surprise are far from eliminated but they are by definition limited by controlled settings. The totality and multiplicity with which the researcher engages in a form of "free play" (Einstein in Schilpp, 1949, p.7) is constrained in advance.

For a theory of principle to be broadly valid, then, it must draw not only on data drawn from the laboratory, but also from less structured, though still disciplined, observations of phenomena "in the wild." Einstein for his part had a wealth of relevant information available to him, both from the laboratory and from the observations of the natural movements of heavenly and earthly bodies. His theories made sense of a "labyrinth of sense impressions", a "totality of empirical fact" (Holton, 1979, p.313, citing Einstein, 1954). This might be something we can easily imagine of Einstein; it's something of a tall order for the rest of us. Nevertheless, we see here a call not only to ambition, but to a re-balancing of our scientific practices, particularly our data production techniques, in order to facilitate the development of better theories of principle. In the context of the wide-ranging disciplinary discussion on the professional practices of research psychologists, our methods and approaches, it behooves us to recognize that the prescriptions for improvements in scientific practice will depend on the question of "improvements for what purpose?" Where a 
need for different forms of theoretical progress is identified, then different kinds of data will need to be produced accordingly, and the more explicit and aware we are of these different aspirations, the better we will manage them.

Several of the criticisms of psychology's poor theoretical state concern the lack of an integrative or over-arching framework (Borsboom, 2013; Muthukrishna \& Henrich, 2019; Wilson, 2011). Muthukrishna and Henrich (2019) explicitly suggest a theory that addresses mechanisms of development and change as offering such a framework, but if Einstein is correct then this is putting the cart before the horse. The encompassing framework is more likely to be, in the first instance, a theory of principle rather than a constructive one - descriptive rather than explanatory.

Where a theory of principle is sought, it becomes important for us to understand the role and value of observations of our phenomena of interest outside of the lab, in the "psychologist-free environment of behavior" (Barker, 1968, p.4; with recognition of the necessary irony that such attempts would involve). If we are to develop descriptive but principled accounts of the structure and pattern of relationships between key aspects of our science, we must seek these patterns in the wild before we can hope to consistently produce them in the laboratory. Where the development of a theory of principle is considered a valid and useful effort for psychology, we will have to spend more time than we have done gathering information on the naturally occurring behavior of human beings in our natural habitat. Before looking at this process in the context of psychology, let us briefly consider its value where it has a richer history, that of biology.

\section{The Value of Observations in the Natural World}

In developing his theories of principle, Einstein (like Newton before him) was able to draw on a wealth of observations and measurements of the phenomena of interest as they unfolded within a natural or uncontrolled environment. In the case of astronomy, for instance, these observations are tabulated by observatories, now with centuries of systematic collection of such data. The world of living systems is much more complex, yet open-minded, sensitive observations of it have played a much-lauded role in the form of natural history (Bates, 1950; Fleischner, 2011).

As with many such phrases, there is no single definition in universal use, though there is a general consensus around the concept in broad terms. Researchers speak, for instance, of a "kind of close, scrupulous observation of nature" (Wilcove \& Eisner, 2000, B24), or "the observation and description of the natural world" (Tewksbury et al., 2014, p.300), "a practice of intentional, focused attentiveness and receptivity to the more-than-human world, guided by honesty and accuracy" (Fleischner, 2011, pp. 5-6). Bates (1950/2014, p.7) describes natural history as "the study of life at the level of the individual - of what plants and animals do, how they react to each other and their environment, how they are organized into larger groupings like populations and communities."

This interest in patterns and relationships, particularly between individuals and their environments, is also an important theme. Wilcover and Eisner (2000, B24) note natural history as involving a "close observation of organisms - their origins, their evolution, their behavior, and their relationships with other species." Tewksbury et al. (2014, p.300) note the study of "organisms and their linkages to the environment being central" to the idea. Sunderland, Klitz, and Yoshihara (2012, p.826) point out that though natural history is often associated simply with the collection of specimens, field notes on context, prevalence, behavior, surrounding vegetation, weather, and landscape, are crucial to the naturalist's practice. The surrounding habitat is considered an essential component of the description of the animal (even if it's a little harder to transport back to the specimen cupboard).

McGann \& Speelman | This is the accepted text. Full paper in press at Theory \& Psychology 
Natural history is a rich practice that provides descriptions of the broader contours and structures within which more mechanistic theories of biological processes are framed. Bartholomew (1986, pp.326-327) claims that "information provided by natural history will always be the touchstone for synthesis (biological significance)", arguing that it is only in the context offered from natural historical accounts that knowledge becomes biological, as opposed to chemical or physical. Sunderland et al. (2014) similarly note that natural history and various experimental and theoretical wings of biological sciences (molecular biology, physiology, morphology, theoretical biology and many others) are mutually dependent.

Among natural history's various aspects, given our interest in the development of theories of principle here, we might focus on two key points:

\section{The cataloging of prevalences and event frequencies in the natural world}

2. The identifying of relationships between different kinds of events, and between organisms and their environments in natural settings.

A range of extant methods might be involved in these two undertakings - from narrative descriptions, to imaging of various kinds, video or audio recording, or the use of observational checklists and similar structured surveys of natural settings. This disciplining of observations forms the backbone to the process of formalization of descriptions of the phenomena that are essential to the development of a theory of principle for a given domain, as Einstein has described it. This is not to say that such theories are built only from observations of the natural world. We have already noted that both Einstein and Newton, for example, also drew from more controlled observations. The richness of naturalistic data is vital, however. As Heft (2018) argues, natural history requires not just an open-minded, descriptive mode, but an ecosystems perspective, one that explicitly records the event or specimen in context in a way that retains important data about the relationship between the phenomenon of interest and the web of related phenomena in which it naturally occurs. While we agree with Heft, it is worth noting that the concept of the ecosystem itself is one of biology's theories of principle, a descriptive and organizing concept that helps structure other investigations. It was derived and refined over time by a series of biologists, most notably Humboldt (Anderson, 2017; Wulf, 2015), but refined by a number of subsequent researchers, typically on the basis of yet more data produced through the process of natural history.

At present, the discipline of psychology largely lacks for such naturalistic data. In the typical story many of us learn as undergraduates, the proper founding of the discipline is taken to be the dedication of laboratory space on either side of the Atlantic in the late 1870's, and there has been a strong emphasis on observation in carefully controlled settings ever since. Historians of psychology have noted that psychologists have standardized around methods that typically value laboratory investigations over work in natural settings (Danziger, 1994; Green, 2015; Stam, 2004). This has been to the extent that Danziger (1994, p.45) describes developments in the early decades of the discipline as a "repudiation of Wundt", who argued vehemently for the need for balance between controlled and naturalistic observations, through the practices of what he termed volkerpsychologie (often translated as cultural, or folk psychology).

The definition of psychology by the founding of laboratories sets the discipline apart from other sciences, where natural history and laboratory practice wove in and out of one another as their methods developed (Anderson, 2017). Indeed, they continue to do so, even as some researchers raise concerns that the emphasis has swung too far away from naturalistic observation (Bartholomew, 1986; Sunderland, Klitz, \& Yoshihara, 2012). The same cannot be said of psychology, which appears 
to have more or less skipped what we might call the "butterfly collection" phase of its scientific development, in our haste to identify ourselves as properly experimental science (Barker, 1968).

Heft $(2001,2018)$ and Morris (2009) point out that though there are some exceptions to this general rule, field work of the kind that can be termed natural history remains very rare, and not very influential within psychological research. This is not to suggest that it does not occur at all, but its role within the discipline is under-valued and unsystematic. Such research tends to already be conducted within already rich theoretical frameworks and has little systematic role in the identification of patterns and relationships proper to natural history (Heft, 2018).

True to the historical development of the science, the majority of psychological researchers begin their careers in the laboratory and tend to stay there. Where research is conducted in natural settings, it is often in the form of intervention or implementation science, such that the kinds of aims served by the activities of natural history cannot be achieved (Heft, 2018).

We argue that this dearth of naturalistic observation is one of the most serious challenges to the emergence of theories of principle, which in turn means that our theoretical sophistication and scientific practice remain weaker than they could and should be.

A natural history of psychology is difficult, but there are some significant positive examples available for us to work with. Descriptive observation of human behavior in the wild is not only possible, it is currently conducted in a number of sub-fields of the discipline in focused ways (examples abound in social psychology, developed from the work of Garfinkel, 1967, Sacks, 1992, and others; naturalistic recording is also relatively well represented in developmental studies, such as Heft, 1979, 1988, Jayaraman, Fausey, \& Smith, 2015; Karasik, Tamis-LeMonda, \& Adolph, 2011; Karasik, Tamis-LeMonda, Adolph, \& Bornstein, 2015; Rossmanith, Costall, Reichelt, López, \& Reddy, 2014). Without question the richest resource for a natural history of psychology is the work of Roger Barker, Louise Shedd Barker, Herbert Wright, Phil Schoggen, and their colleagues, who conducted more than twenty-five years of field work in a small town in rural Kansas, work that is as impressive as it is unknown.

In a reflection at the end of his career Barker (1979) described an experience of shock, a "negative 'Aha!'” (p.2150), during a journey as the train he was on stopped in numerous small towns through rural Illinois in the United States. Despite being an expert in human behavior he had little idea beyond his own common sense as to what actually happened, day in, day out, in these various places. Moving to the University of Kansas, he, Louise Shedd Barker, a zoologist, and a cadre of graduate students, set up a field research station in the town of Oskaloosa (referred to in their research as "Midwest"). Over a period of twenty-five years (from 1948 to 1973, when the Midwest Psychological Field Station closed), they went from observing every activity of a small group of children, to recording data on many different settings of people's activities within the public spaces of the town, as well as comprehensive assessments of the various resources the town provided for those activities. The result is a striking body of work which identifies some deeply important relationships between human behavior and physical and social environments in which they take place.

It is beyond the scope of this paper to offer anything other than a few breadcrumbs to lead the reader to this work (see Heft, 2001, Chapter 7; Barker, 1968; Schoggen, 1989), though the primary results of it, the theory of behavior settings, is perhaps a prime candidate for a theory of principle for behavior. Years of extensive recording and cataloging of behavior enabled the group to identify patterns, relationships, and structures that offered significant insights into psychology. For the 
moment, suffice it to point out that if we have an interest in predicting behaviors, and have access only to a single piece of information about a person, then by far the most valuable datum we can seek is "what setting are they in?" In an oft-used phrase to summarize the idea "When in church, people behave church; in school, people behave school." Behavior tends to vary more within people across settings, than between people within settings.

Psychological researchers have a tendency, remarked upon by Barker (1968, p.4) and largely unchanged since, to either take complete control of the environment (through the provision of stimulus packages in blandly painted and sparely furnished laboratories) or treat the environment as being probabilistically structured, a statistical puzzle to solve. These are understandings of the relationship between people and their environments that do not survive an effort after natural history, and observation of the highly structured, nested character of settings for behavior in the human habitat.

Barker and his colleagues developed a rich body of theory derived from their observational research, one that offers substantial return on the investment in getting to know it. But it is work that has seen little replication, and surprisingly sparse study by researchers outside of the original research group. We should note explicitly, however, that some of the Midwest work, particularly that with regards to the population (and under-population) of behavior settings has been examined by others (e.g. Wicker, 1978), but the extensive naturalistic data gathering remains an outlier in the field. This appears to have been due to a number of factors, but largely lack of available funding and constraints of professional incentives which run counter to slow careful descriptive work (Scott, 2005).

The Midwest team did not set out to develop a theory of principle. Their data collection efforts were not all purely descriptive, and in making sense of the dynamics of behavior settings they deploy a host of accounts of mechanism and other characteristic of constructive theories. Both early and later articulations of the theory include reference to "circuits" of various kinds that initiate, progress, and maintain the setting during its existence. The discovery of behavior settings in and of itself, however, is the advancement of a theory based on what Barker described as "atheoretical, phenomena-centered" (1978, p.46) observations, not built from the hypothesized components of individual human behavior. The mechanisms advanced by Barker (1968), Schoggen (1989) and others comes subsequent, constructive theories attempting to respond to the demands of the principle theory.

The team's various publications provide extensive information on their methods of observation, recording, and analysis, but all conducted in a small rural town, and with an emphasis on the "molar" level of description - behavior in its contours rather than its fine details, a decision made for reasons of practicality and to address an apparent gap in psychological research generally (Barker, 1968). These methods offer the potential for the creation of rich and important corpuses of behavioral data, but only if the value and purpose of it can be imagined by psychological scientists and their funders. It is slow work, demanding substantial investments of time in particular. Modern recording technologies will no doubt allow for some work to be divided in ways it has typically not been, but only if we can manage to pick our way carefully through the ethical challenges and legal complications of doing so. It is worth remarking, though, that these are the primary obstacles for the conduct of research that can in principle be conducted with nothing more than patience, care, a notebook, and pencil.

We have noted that one purpose of natural history is to provide descriptions of the naturally occurring phenomena of a scientific domain. Natural histories enable scientists to understand the 
relative frequency of particular kinds of occurrences, in order to evaluate how representative their controlled studies are of ordinarily occurring events (Bartholomew, 1986; Bates, 1950; Brunswik, 1947 , makes similar observations relevant to psychology). This would also mean for psychology developing a clearer picture of the diversity, the range and variability, of behavior and mental life, something it is surprisingly difficult to do from our extant body of theory (see the preface to Barker et al, 1978, for an enlightening outlining of this issue). Additionally, natural histories can be conducted at varying levels of description (somewhat in contrast to laboratory experiments, where the focus is on a very narrow range of temporal and spatial scales for behavior). In doing so, they allow us to look at not just the relative frequency, but the relationships in time and space, between different phenomena of interest to the discipline as they occur in the environment not under the control of the psychologist.

For instance, a natural history of cognition would enable us to form descriptions, and then hypotheses, about the importance of places in the emergence and development of cognitive functions in normal settings (Barker, 1968; Klein, 1993; Schoggen, 1989). It would also enable the examination of patterns of behaviors (cognitive, social, emotional), over the course of minutes, hours, days, or longer, which could offer us opportunities to perceive, hypothesize, and ultimately test theories about, relationships between different aspects of behavior or mental activity.

We believe that a more refined understanding of different kinds of theory and their complementary roles in the development of science offers one way to articulate the importance of such efforts. Alas, our discipline's blindness to distinctions between, and distinct roles of, different kinds of theory offers a somewhat pessimistic outlook for such work to be undertaken. We believe that this leaves psychologists trying to do science with one arm tied behind their backs - addressing questions of data collection and theory building from only one aspect of a complementary set of perspectives.

\section{Principles, Constructions, Explorations, Confirmations}

In the past year more vocal critics have warned that the methodological and analytical changes that have drawn the majority attention on this issue (such as registered reports and more extreme critical values for statistical hypothesis testing) will not by themselves suffice to address the problems of scientific progress in psychological science. We welcome this more explicit discussion about the standards of our theories. It is in agreement with those researchers bringing these considerations to a wider audience that we note in this paper the importance of being aware of the different kinds of roles that theories play in the development of scientific knowledge, and the varying criteria that are appropriate in the evaluation of distinct forms of theory.

In positioning the present paper within this discussion, however, we should note carefully where are arguments resonate, and where they differ from others'. Specifically, a set of related-seeming distinctions have been deployed to get to grips with the theoretical import (or lack thereof) of certain reforms in psychological science. We wish to be clear in how our work relates, and does not, to this existing debate. If we simplify the difference between principle and constructive theories, we find a distinction that is readily familiar to psychologists, and anyone else versed in the literature on reforms in scientific practice over the past few years. With an emphasis on observation and openness to novel patterns, a caricature of theories of principle might seem like "exploratory research", with the sharply focused and hypothesis driven constructive theories being "confirmatory research". This mapping is not quite right, however, and should be resisted.

In the first instance, exploratory and confirmatory research are typically defined not by their rela- 
tionship to particular forms of theory, but by a narrow set of methodological practices, involving the prior specification of hypotheses and modes of analysis (Wagenmakers, Dutilh \& Sarafoglou, 2018; Wagenmakers et al., 2012). The value of this distinction for theory building has been called into question (Oberauer \& Lewandowsky, 2019; Szollosi \& Donkin, 2019), as the key variables that affect the value of data for evaluation of a theory have little to do with the plans of the researcher, and more to do with the logical structure of the theory and its relationship to the observations in question. Though there is merit in the disciplining of data production and analytic practices, this methodological distinction neither fixes the limitations of much psychological research, nor allows for the kinds of division of labor and complementarity of effort that underlies the principle-constructive distinction.

Oberauer and Lewandowsky (2019) offer a somewhat different pair of categories in the notion of discovery-oriented vs. theory-testing research. Rather than a dichotomy, however, they suggest this is a continuum governed not by the specific reporting practices of the scientists, but the prior probability of a given theory, and the probability of a theory being true given a particular set of observations. In the case of theory-testing research, the hypotheses in question are logically entailed by the theory being examined. There is a strong logical dependence between them such that the implications of the observations made in testing the hypotheses bear directly on the structure or parameters of the theory. In the case of discovery-oriented research the logical relationship is looser. Oberauer and Lewandowsky (2019) describe hypotheses in discovery-oriented research being motivated, rather than entailed, by the theory. The theory is much less likely to stand or fall on the observations made, and is considered much more flexible in response to those observations.

Though the discovery-oriented vs. theory-testing distinction gets at the relationship between theory and data, this remains a difference of observational or experimental practice. This continuum of difference could apply to either theories of principle or constructive theories, and does not (nor is it intended to) capture the kind of inter-dependency of different kinds of knowledge that Einstein's description illustrates. Theories of principle are without question the products of exploratory research, but those explorations are not conducted through a series of statistical hypothesis tests. It may well be that statistical methods of various kinds will help us bootstrap our recognition of patterns, be part of a set of tools that might enable those of us who fall well short of being a Newton or Einstein to find some of those important relationships. But the tests, howsoever disciplined, will not be able to completely replace the abductive logic needed. Indeed, the data of natural history that we have advocated for here are less concerned with central tendencies, and more with variation and individualities (Bartholomew, 1986). To make progress on this issue we will simply have to recognise the value of different modes of theory, and purposefully undertake programs of natural historical research, and careful consideration of the data produced.

\section{Conclusion: Data follow theories follow data}

Theory is the formal structuring of knowledge according to our best understanding of phenomena in which we are interested. The advancement of scientific knowledge is a matter of the continuous refinement of theory such that it enables better coordination with the world we experience.

Advancement of knowledge is not a unitary phenomenon, however. It is not a slow march of progress from ignorance to enlightenment. It is more like way-finding within a complex landscape of ignorance, certainty, and value; not measured on a single dimension. It is neither simply the improving accuracy of theories, nor the increasing confidence in the results of statistical tests. Advancement is multidimensional, depending on the kind of understanding that theories provide,

McGann \& Speelman | This is the accepted text. Full paper in press at Theory \& Psychology 
and the multifarious values of these different theories for different purposes. We have outlined in this paper just two different kinds of theories, but also that these different theories have different relationships with data, and tend to give differential roles to different kinds of data. Calibrating our data production practices with theory is thus not a linear process, but one with a feedback loop. Best methodological practice will depend on our understanding of theories, and those theories will thus shift as we continued to generate and interpret different data. We must take great care to avoid any simple hierarchy of priorities. Reforms of methodological practice must make reference to the theories that frame them, while calls for improvement in theory must similarly situate themselves within questions of method.

In this paper we argue that the discipline of psychology, if it can be coherent at all, must begin to reemphasize the importance of a natural history of behavior and experience, one that acknowledges diversity and relationships in addition to norms and averages. Modern psychology would have some consolation to offer Roger Barker experiencing his negative epiphany on a train journey through 20th century Illinois, though perhaps much less than we should.

We celebrate the number of psychologists embracing the urgency of reform like never before in the discipline. We also acknowledge, however, the importance of an open and critical dialogue around even the most fundamental tenets of that reform, and we urge psychologists to consider carefully the circular, inter-dependent relationship between theory and practice in the context of a diversity of theories, and diversity of practices.

Acknowledgements This paper owes a great deal to extensive comments and feedback from Berna Devezer, and also from Harry Heft, whose suggestions made significant contributions to the structure and content of the final version.

\section{References}

Anderson, J. G. T. (2017). Why ecology needs natural history. American Scientist, 105(5), 290-297. https://doi.org/10.1511/2017.105.5.290

Balashov, Y., \& Janssen, M. (2003). Presentism and relativity. The British Journal for the Philosophy of Science, 54(2), 327-346. https://doi.org/10.1093/bjps/54.2.327

Barker, R. G. (1968). Ecological psychology: Concepts and methods for studying the environment of human behavior. Stanford University Press.

Barker, R. G. (1979). Settings of a professional lifetime. Journal of Personality and Social Psychology, 37(12), 2137-2157. https://doi.org/10.1037/0022-3514.37.12.2137

Barker, R. G. (1978). Habitats, environments, and human behavior. Jossey-Bass Incorporated Pub.

Barrows, C. W., Murphy-Mariscal, M. L., \& Hernandez, R. R. (2016). At a crossroads: The nature of natural history in the twenty-first century. BioScience, 66(7), 592-599.

Bartholomew, G. A. (1986). The Role of Natural History in Contemporary Biology. BioScience, 36(5), 324-329. https://doi.org/10.2307/1310237

Borsboom, D. (2013, November 20). Theoretical Amnesia. Retrieved November 19, 2018, from Open Science Collaboration Blog website: http://osc.centerforopenscience.org/2013/11/20/theoreticalamnesia/ 
Botkin, D. B., Saxe, H., Araujo, M. B., Betts, R., Bradshaw, R. H., Cedhagen, T., .. Faith, D. P. (2007). Forecasting the effects of global warming on biodiversity. Bioscience, 57(3), 227-236. https://doi.org/10.1641/B570306

Brunswik, E. (1947). Systematic and representative design of psychological experiments. In J. Neyman (Ed.), Proceedings of the Berkeley symposium on mathematical statistics and probability (pp. 143-202). University of California Press.

Carsel, T., Demos, A. P., \& Motyl, M. (2018). Strong scientific theorizing is needed to improve replicability in psychological science. Behavioral and Brain Sciences, 41. https://doi.org/10.1017/S0140525X1800078X

Danziger, K. (1994). Constructing the subject: Historical origins of psychological research. Cambridge University Press.

Dar, R. (1987). Another look at Meehl, Lakatos, and the scientific practices of psychologists. American Psychologist, 42(2), 145-151. https://psycnet-apa-org/doi/10.1037/0003-066X.42.2.145

Dewsbury, D. A. (2009). Is psychology losing its foundations? Review of General Psychology, 13(4), 281-289. https://doi.org/10.1037/a0017760

Einstein, A. (1919, November 28). What is the theory of relativity? London Times, pp. 13-14.

Felline, L. (2011). Scientific explanation between principle and constructive theories. Philosophy of Science, 78(5), 989-1000. https://doi.org/10.1086/662270

Fleischner, T. L. (2011). The mindfulness of natural history. In T. L. Fleischner (Ed.), The way of natural history. Trinity University Press.

Flores, F. (1999). Einstein's theory of theories and types of theoretical explanation. International Studies in the Philosophy of Science, 13(2), 123-134. https://10.1080/02698599908573613

Garfinkel, H. (1967). Studies in ethnomethodology. Englewood Cliffs, NJ: Prentice-Hall.

Green, C. D. (2015). Why psychology isn't unified, and probably never will be. Review of General Psychology, 19(3), 207-214. https://doi.org/10.1037/gpr0000051

Heft, H. (1979). Background and focal environmental conditions of the home and attention in young children. Journal of Applied Social Psychology, 9(1), 47-69. https://doi-org/10.1111/j.15591816.1979.tb00794.x

Heft, H. (1988). Affordances of children's environments: A functional approach to environmental description. Children's Environments Quarterly, 29-37. https://doi-org/10.1111/j.1559-1816.1979.tb00794.x

Heft, H. (2001). Ecological psychology in context: James Gibson, Roger Barker, and the legacy of William James's radical empiricism. (1st ed.). London: Lawrence Erlbaum Associates.

Heft, H. (2018). Places: Widening the Scope of an Ecological Approach to Perception-Action With an Emphasis on Child Development. Ecological Psychology, 30(1), 99-123. https://doi.org/10.1080/10407413.2018.

Holton, G. (1979). Constructing a theory: Einstein's model. The American Scholar, 309-340. https://www.jstor.org/st

Jayaraman, S., Fausey, C. M., \& Smith, L. B. (2015). The Faces in Infant-Perspective Scenes Change over the First Year of Life. PLOSONE, 10(5), e0123780. https://doi.org/10.1371/journal.pone.0123780 
Karasik, L. B., Tamis-LeMonda, C. S., \& Adolph, K. E. (2011). Transition from crawling to walking and infants' actions with objects and people. Child Development, 82(4), 1199-1209. https://doiorg/10.1111/j.1467-8624.2011.01595.x

Karasik, L. B., Tamis-LeMonda, C. S., Adolph, K. E., \& Bornstein, M. H. (2015). Places and postures: A cross-cultural comparison of sitting in 5-month-olds. Journal of Cross-Cultural Psychology, 46(8), 1023-1038. https://doi.org/10.1177/0022022115593803

Lange, M. (2014). Did Einstein Really Believe that Principle Theories are Explanatorily Powerless? Perspectives on Science, 22(4), 449-463.

Morris, E. K. (2009). Behavior Analysis and Ecological Psychology: Past, Present, and Future. a Review of Harry Heft's Ecological Psychology in Context. Journal of the Experimental Analysis of Behavior, 92(2), 275-304.

Muthukrishna, M., \& Henrich, J. (2019). A problem in theory. Nature Human Behaviour, 3(3), 221. https://doi.org/10.1038/s41562-018-0522-1

Oberauer, K., \& Lewandowsky, S. (2019). Addressing the theory crisis in psychology. Psychonomic Bulletin \& Review. https://doi.org/10.3758/s13423-019-01645-2

Rossmanith, N., \& Reddy, V. (2016). Structure and Openness in the Development of Self in Infancy. Journal of Consciousness Studies, 23(1-2), 237-257. https://doi.org/10.3389/fpsyg.2014.01390

Sacks, H. (1992). Lectures on conversation, vols I and II (edited by G. Jefferson with introductions by E.A. Schegloff). Blackwell.

Schilpp, P. A. (Ed.). (1949). Albert Einstein: Philosopher-scientist. The Library of Living Philosophers.

Schoggen, P. (1989). Behavior settings: A revision and extension of Roger G. Barker's "Ecological Psychology." Stanford University Press.

Scott, M. M. (2005). A powerful theory and a paradox ecological psychologists after Barker. Environment and Behavior, 37(3), 295-329. https://doi.org/10.1177/0013916504270696

Stam, H. J. (2004). Unifying psychology: Epistemological act or disciplinary maneuver? Journal of Clinical Psychology, 60(12), 1259-1262. https://doi-org/10.1002/jclp.20069

Sunderland, M. E., Klitz, K., \& Yoshihara, K. (2012). Doing natural history. BioScience, 62(9), 824-829. https://doi.org/10.1525/bio.2012.62.9.8

Szollosi, A., \& Donkin, C. (2019a). Arrested theory development: The misguided distinction between exploratory and confirmatory research [Preprint]. https://doi.org/10.31234/osf.io/suzej

Szollosi, A., \& Donkin, C. (2019b). Neglected sources of flexibility in psychological theories: from replicability to good explanations. Computational Brain \& Behavior. https://doi.org/10.1007/s42113019-00045-y

Tewksbury, J. J., Anderson, J. G. T., Bakker, J. D., Billo, T. J., Dunwiddie, P. W., Groom, M. J., ... Wheeler, T. A. (2014). Natural History's Place in Science and Society. BioScience, 64(4), 300-310. https://doi.org/10.1093/biosci/biu032

Wagenmakers, E.-J., Dutilh, G., \& Sarafoglou, A. (2018). The Creativity-Verification Cycle in Psycho- 
logical Science: New Methods to Combat Old Idols. Perspectives on Psychological Science, 13(4), 418-427. https://doi.org/10.1177/1745691618771357

Wagenmakers, E.-J., Wetzels, R., Borsboom, D., van der Maas, H. L. J., \& Kievit, R. A. (2012). An Agenda for Purely Confirmatory Research. Perspectives on Psychological Science, 7(6), 632-638. https://doi.org/10.1177/1745691612463078

Wilcove, D. S., \& Eisner, T. (2000). The impending extinction of natural history. The Chronicle of Higher Education; Washington, 47(3), B24.

Wilson, A. (2011). Theory, and why it's time psychology got one. Retrieved October 13, 2017, from Notes from Two Scientific Psychologists website: http://psychsciencenotes.blogspot.com/2011/11/theoryand-why-its-time-psychology-got.html

Wulf, A. (2015). The invention of nature: Alexander von Humboldt's new world. John Murray. 